\title{
On the convergence of nonlinear modes of a finite element model
}

\author{
Ramesh Balagangadhar ${ }^{\mathrm{a}}$ and Joseph C. Slater ${ }^{\mathrm{b}, *}$ \\ ${ }^{a}$ Goodrich Aerospace, Engineering Technologies, 101 Waco St, Troy, OH 45373, USA \\ ${ }^{\mathrm{b}}$ Department of Mechanical and Materials Engineering, Wright State University, Dayton, OH 45435, USA
}

\begin{abstract}
Convergence of finite element models is generally realized via observation of mesh independence. In linear systems invariance of linear modes to further mesh refinement is often used to assess mesh independence. These linear models are, however, often coupled with nonlinear elements such as CFD models, nonlinear control systems, or joint dynamics. The introduction of a single nonlinear element can significantly alter the degree of mesh refinement necessary for sufficient model accuracy. Application of nonlinear modal analysis [1,2] illustrates that using linear modal convergence as a measure of mesh quality in the presence of nonlinearities is inadequate. The convergence of the nonlinear normal modes of a simply supported beam modeled using finite elements is examined. A comparison is made to the solution of Boivin, Pierre, and Shaw [3]. Both methods suffer from the need for convergence in power series approximations. However, the finite element modeling method introduces the additional concern of mesh independence, even when the meshing the linear part of the model unless p-type elements are used [4]. The importance of moving to a finite element approach for nonlinear modal analysis is the ability to solve problems of a more complex geometry for which no closed form solution exists. This case study demonstrates that a finite element model solution converges nearly as well as a continuous solution, and presents rough guidelines for the number of expansion terms and elements needed for various levels of solution accuracy. It also demonstrates that modal convergence occurs significantly more slowly in the nonlinear model than in the corresponding linear model. This illustrates that convergence of linear modes may be an inadequate measure of mesh independence when even a small part of a model is nonlinear.
\end{abstract}

\section{Nomenclature}

$\mathbf{u} \quad$ Displacement vector, physical coordinates

v Velocity vector, physical coordinates

$\mathbf{r} \quad$ Displacement vector, linear modal coordinates

s Velocity vector, linear modal coordinates

$K \quad$ Stiffness matrix

[M] Mass matrix

$[\Lambda] \quad$ Matrix of eigenvalues

$[S] \quad$ Matrix of mode shapes

$A \quad$ Cross sectional area

$E \quad$ Young's modulus

I Second moment of area

*Corresponding author. E-mail: joseph.slater@wright.edu. 


$\begin{array}{ll}L & \text { Length of beam } \\ m & \text { Mass per unit length } \\ x & \text { Abscissa along beam } \\ u(x, t) & \text { Transverse deflection of beam } \\ (\cdot s) & \text { Derivative wrt } s \\ (\cdot) & \text { Time derivative } \\ \delta & \text { Dirac delta function } \\ \gamma & \text { Nonlinear stiffness coefficient } \\ \rho & \text { Density }\end{array}$

\section{Introduction}

Vibration of linear systems is governed by a set of linear ordinary differential equations. The solutions of linear differential equations are obtained most conveniently by means of linear transformations rendering the set of equations independent. The concept of normal modes and of eigenvalues is well defined for such systems. A linear combination of the normal solutions yields the total solution of the system. For linear, conservative, non-gyroscopic, spatially distributed systems each normal mode has associated with it a mode shape, mathematically represented by an eigenfunction, and a natural frequency represented by the corresponding eigenvalue. These normal modes represent a special set of motions of the system in which it behaves like a system of lower order. In linear vibratory systems, the dynamics of each individual mode is governed by a second order linear modal oscillator which is uncoupled from all other modal oscillators.

The study of the modal vibrations of nonlinear systems with many degrees of freedom is concerned with the search for some or all periodic solutions of systems of nonlinear differential equations. Meirovitch [5] defines normal modes in terms of linear eigenfunctions and the expression of an arbitrary system response as a superposition of linear modal responses. The concept of linear normal modes of motion is well developed for a wide class of dynamic systems via linearization of the nonlinear equations of motion about an equilibrium configuration to obtain linear differential equations [6]. However, this approach is not generally valid for large amplitudes of motion.

The concept of nonlinear normal modes for $n$-degree of freedom nonlinear systems was first defined in a general manner by Rosenberg [7]. In his works, Rosenberg considers the existence and stability of normal modes for a system of $n$ masses interconnected by nonlinear symmetric springs and having $n$ degrees of freedom. Subsequently, determination of nonlinear normal modes has been accomplished via an asymptotic approach [8], energy approaches [9], the method of multiple scales [10], and Poincaré maps [11]. Shaw and Pierre [2] generalize the definition of normal modes of nonlinear systems to include non-conservative, gyroscopic, and continuous systems. Methodologies developed by Shaw and Pierre [1,2,12], Shaw [13] and Boivin, Pierre, and Shaw [3] illustrate the use of the method of invariant manifolds to obtain normal modes for weakly nonlinear systems and demonstrate a means of generating differential equations of motion that govern the dynamics of a system undergoing a nonlinear modal motion. These methods use procedures based on center manifold theory [14]. Two different approaches based on invariant manifold methodologies to determine the nonlinear modes of systems have been demonstrated. The first treats (continuous) systems as such and obtains the nonlinear normal modes directly from the differential equations of motion [12]. This is referred to by the authors as the continuous nonlinear normal mode method. The second discretizes the system's equations of motion using its linear normal modes, and then applies the theory developed for nonlinear discrete systems and is referred to as the discretized nonlinear normal mode method. A variation of this is demonstrated by Nayfeh and Nayfeh [10] where instead of using real variables, a complex variable approach is used. In [3], Boivin, Pierre, and Shaw show the continuous approach to be less reliable than the discretized approach. The task of presenting a comprehensive survey of the various nonlinear modal methodologies and results is beyond the scope of this relatively brief contribution. The works of Vakakis [15] and Nayfeh [16] illustrate the vast range methodologies that have been applied to the solution for nonlinear modes. Apiwattanalunggarn, Shaw, Pierre and Jiang[17] applies nonlinear modal methods to a finite element model to generate single mode reduced order models. Pesheck, Pierre and Shaw[18] demonstrate the application of co-ordinate transformations along with Galerkin procedures to generate highly accurate manifold models. 


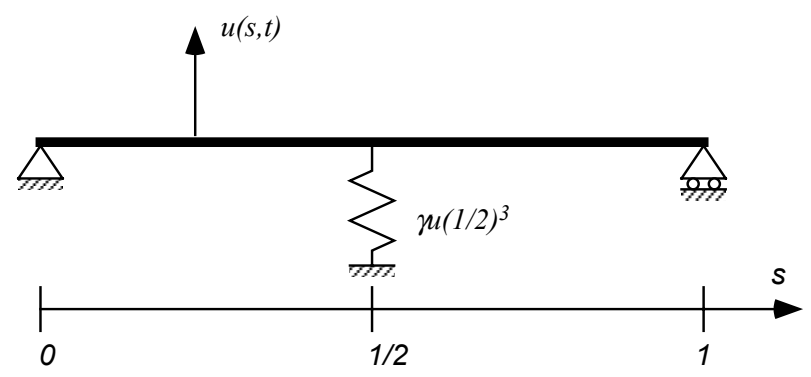

Fig. 1. Euler Bernoulli beam with cubic spring.

Here the invariant manifold method presented by Shaw and Pierre [1,2,12] and Shaw [13] is used to determine the nonlinear normal mode shapes of a finite element model of a beam with a single nonlinear element added. The work illustrates on a relatively simple system how the introduction of just a single nonlinearity can have a significant effect on the degree to which the linear mesh must be refined. The term normal is used loosely here in that nonlinear modes are generally only normal in the immediate vicinity of equilibrium. This approach provides a means of constructing mode shapes and modal oscillators for nonlinear systems in the immediate vicinity of equilibrium. Rather than view nonlinear normal modes as synchronous motions of conservative systems, they are viewed as motions on invariant manifolds that are tangent to, and of the same dimension as, the linear eigenspaces in the system state space [2]. The convergence study performed here does not depend specifically on the method used, however, as each of the aforementioned techniques can be demonstrated to yield functionally equivalent results for undamped non-gyroscopic systems.

Difficulties in obtaining closed-form solutions to continuous (distributed parameter) systems can be attributed to the inherent difficulty of solving partial differential equations, with or without space dependent coefficients, and in satisfying the boundary conditions. These difficulties can be circumvented by eliminating the spatial dependence of the problem using numerical techniques. Prior work on continuous systems in the literature has focused on the solution of problems for which the linearized problem has a closed-form solution. The ability to solve practical engineering problems this way is inhibited by the inherent geometrical complexity of engineering structures. This work focuses on the application of the finite element method as a general discretization procedure and considers the impact of discretization on nonlinear normal mode error. The finite element method is applied here to the problem presented by Boivin, Pierre, and Shaw [3] using the general method developed by Shaw and Pierre [1,2] and extended by Shaw and Pierre [1], Shaw [13] and Boivin, Pierre, and Shaw [3] to demonstrate the convergence of a finite element model. Results are compared to the converged results presented by Boivin, Pierre, and Shaw [3] using a continuous solution. Convergence as a function of the level of discretization is presented yielding some unexpected results.

\section{Finite element model}

The system studied is a simply-supported, linear Euler-Bernoulli beam of constant cross section with a nonlinear cubic spring attached at the center analyzed by Boivin, Shaw and Pierre [3] (Fig. 1). If the spring is chosen to be purely cubic and the beam is assumed to deform in the linear range, then the normal modes of the linearized system will be the same as those of a simply supported beam (i.e. pure sine waves). Most of the symbolic manipulation and almost all of the numerical work for the examples was done using Mathematica ${ }^{\circledR}$. The plots were generated using MATLAB ${ }^{\circledR}$.

The continuous equation of transverse motion for a beam of unit length is

$$
m \ddot{u}+E I u_{s s s s}+\gamma u^{3} \delta\left(s-\begin{array}{l}
1 \\
2
\end{array}\right)=0, \quad s \in[0,1]
$$

Dividing the equation of motion Eq. (1) by $m$ reduces this to a pseudo-non-dimensional form, 


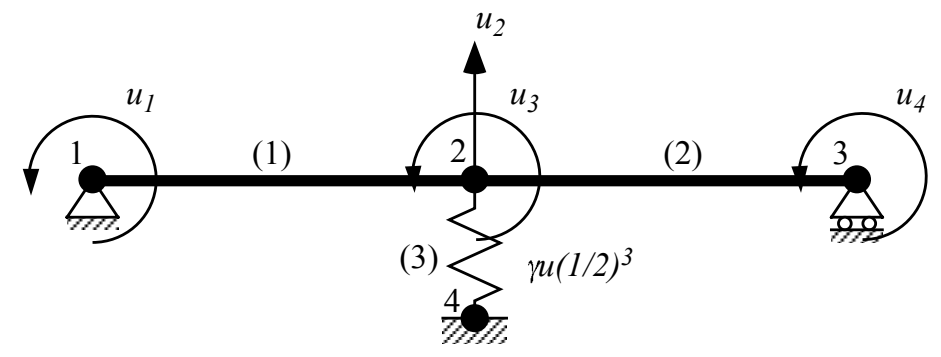

Fig. 2. Finite element model of the beam with cubic spring.

$$
\ddot{u}+\alpha u_{s s s s}+\beta u^{3} \delta\left(s-\begin{array}{l}
1 \\
2
\end{array}\right)=0, \quad s \in[0,1]
$$

where $\alpha=E I / m$ and $\beta=\gamma / m$. The boundary conditions are given by

$$
\begin{array}{ll}
u(0)=0, & u_{s s}(0)=0 \\
u(1)=0, & u_{s s}(1)=0
\end{array}
$$

We now apply the finite element method to the above problem. The beam is divided into a varying number $(2,4,8,16)$ of elements and the equations of motion of the discretized system are obtained. The results of the analysis will demonstrate that the linear part of the problem must be modeled more accurately when significant nonlinearity exists than would traditionally be necessary for a purely linear system. The procedure is demonstrated here for the first and third modes of a two element model. Four, eight and sixteen element formulations are developed in a similar manner with the results of all four analyses presented later.

\subsection{Two-element formulation}

After the boundary conditions have been applied, the beam model has four degrees of freedom (Fig. 2). Nodes 1 and 3 have one rotational degree of freedom each and no translational degrees of freedom as a result of the boundary constraints.

The mid-beam node, 2 , has both rotational and translational degrees of freedom and is also shared by the nonlinear spring.

\subsection{Finite element solution}

The matrix form of the linear equations of motion of the beam modeled using standard Euler-Bernoulli elements in physical coordinates are [19]

$$
M \ddot{\mathbf{u}}+K \mathbf{u}=\mathbf{0}
$$

where $M$ and $K$ are the mass and stiffness matrices of the beam. The eigenvectors and eigenvalues of the normalized linear system, assuming $\alpha=1$, are the eigenvalues and eigenvectors of the matrix,

$$
A=M^{-1} K
$$

The eigenvectors are

$$
S=\left[\begin{array}{cccc}
-1 & 1 & 1 & 1 \\
-0.318 & 0 & 0 & 0.0546 \\
0 & -1 & 1 & 0 \\
1 & 1 & 1 & 1
\end{array}\right]
$$


and the eigenvalues of the system are,

$$
\Lambda=\operatorname{diag}\{98.1795,1920 ., 40320 ., 12131 .\}
$$

Incorporating the nonlinearity of the beam into the system changes the global equations of motion to

$$
M \ddot{\mathbf{u}}+K \mathbf{u}+\left[\begin{array}{c}
0 \\
\gamma u_{2}^{3} l^{3} \\
E I \\
0 \\
0
\end{array}\right]=\mathbf{0}
$$

Consider the coordinate transformation

$$
\mathbf{u}=S\{\mathbf{r}(t)\},
$$

where $\{\mathbf{r}(t)\}$ is the column vector of modal coordinates and $S$ is the transformation matrix that consists of the eigenvectors of the linear problem. Substituting for $\mathbf{u}$ and premultiplying Eq. (8) by $S^{T}$ results in the following four linearly decoupled, but nonlinearly coupled, equations:

$$
\begin{aligned}
& \ddot{r}_{1}+98.1795 r_{1}+0.205978 \beta r_{1}^{3}-0.106064 \beta r_{1}^{2} r_{3}+0.0182052 \beta r_{1} r_{3}^{2}-0.0010416 \beta r_{3}^{3}=0 \\
& \ddot{r}_{2}+1920 r_{2}=0 \\
& \ddot{r}_{3}+12130.7 r_{3}-0.980562 \beta r_{1}^{3}+0.50492 \beta r_{1}^{2} r_{3}-0.086666 \beta \beta r_{1} r_{3}^{2}+0.00495854 \beta r_{3}^{3}=0 \\
& \ddot{r}_{4}+40320 r_{4}=0
\end{aligned}
$$

where $\beta=\gamma / \rho A=\gamma / m$. Note that if $\beta$ is set to zero these equations reduce to the four linear modal equations. As in reference [3], $\beta$ has been set to $1 \times 10^{4}$.

This set of coupled, nonlinear ordinary differential equations describes the dynamics of the linear modal components of the motion. The second and fourth equations that are now decoupled represent the modal equations for the even modes two and four. The solution of the decoupled equations is straightforward and is not presented with here. The other two equations remain coupled in the nonlinear terms and represent the linear modal equations of modes one and three. The nonlinear terms represent nonlinear modal coupling that exists after a traditional linear modal analysis is performed. These equations can be numerically integrated using a fourth order accurate Runge-Kutta time marching scheme to obtain what can be considered the "exact" dynamics of the system.

One can obtain approximations of the nonlinear modes of the system by applying to this set of nonlinear ordinary differential equations the general method developed by Shaw and Pierre [2]. To obtain the first nonlinear mode shape we assume $r_{1}$ (the first linear modal displacement) to be the equivalent to modal the coordinate $u$. A normal mode motion is assumed by requiring that $r_{1}, \dot{r}_{1}, r_{3}$ and $\dot{r}_{3}$ are related to the modal displacement $u$ and modal velocity $v$ as

$$
\begin{aligned}
& r_{1}=u \\
& \dot{r}_{1}=v \\
& r_{3}=R_{3}(u, v)=a_{1} u+a_{2} v+a_{3} u^{2}+a_{4} u v+a_{5} v^{2}+a_{6} u^{3}+a_{7} u^{2} v+a_{8} u v^{2}+a_{9} v^{3}+\ldots \\
& \dot{r}_{3}=S_{3}(u, v)=b_{1} u+b_{2} v+b_{3} u^{2}+b_{4} u v+b_{5} v^{2}+b_{6} u^{3}+b_{7} u^{2} v+b_{8} u v^{2}+b_{9} v^{3}+\ldots
\end{aligned}
$$

where the coefficients $a_{1}, b_{1}, a_{2}, b_{2}, \ldots$ are to be determined.

Taking the time derivative of $R_{3}$ and $S_{3}$ and applying the chain rule yields

$$
\dot{R}_{3}=a_{1} \dot{u}+a_{2} \dot{v}+2 a_{3} u \dot{u}+a_{4} u \dot{v}+a_{4} \dot{u} v+2 a_{5} v \dot{v}+\ldots
$$

and

$$
\dot{S}_{3}=b_{1} \dot{u}+b_{2} \dot{v}+2 b_{3} u \dot{u}+b_{3} u \dot{v}+b_{3} \dot{u} v+2 b_{5} v \dot{v}+\ldots
$$


The equations of motion are also expressed in $u$ and $v$ by substitution of Eq. (11) as,

$$
\begin{aligned}
& \ddot{r}_{1}=\dot{s}_{1}=-98.1795 u-2059.78 u^{3}+1060.64 u^{2} R_{3}(u, v)-182.052 u R_{3}^{2}(u, v)+10.416 R_{3}^{3}(u, v) \\
& \ddot{r}_{3}=\dot{s}_{3}=9805.62 u^{3}-12130.7 R_{3}(u, v)-5049.2 u^{2} R_{3}(u, v)+866.66 u R_{3}^{2}(u, v)-49.5854 R_{3}^{3}(u, v)
\end{aligned}
$$

By definition,

$$
\begin{aligned}
& \dot{R}_{3}=S_{3} \\
& \dot{S}_{3}=\ddot{r}_{3}
\end{aligned}
$$

Equations (12) and (13) are now used to replace $\dot{R}_{3}$ and $\dot{S}_{3}$, and $S_{3}$ and $\ddot{r}_{3}$ are replaced using the expressions of Eqs (11d) and (15). Finally, $\dot{u}$ is replaced by $v$ in the resulting expressions, and $\dot{v}$ is replaced using Eq. (12) (Recall $\left.v=\dot{r}_{1}\right)$. This yields the required conditions for a modal motion. The resulting equations are then expanded to cubic order in $u$ and $v$, and the coefficients of $u, v, u^{2}, u v, v^{2}, u^{3}, u^{2} v, u v^{2}$, and $v^{3}$ are gathered to provide the equations for the $a_{j}$ and $b_{j}$ coefficients. (The right side is subtracted from the equation in order to give equations that are equal to zero). There are two equations for each term, one from each of the two Eq. (16) than be solved for the coefficients in Eqs (11c) and (11d). We can now express $r_{4}$ in terms of the modal coordinate $r_{3}$. Using Eq. (11c) and the results obtained for the coefficients $a_{1}, b_{1}, a_{2}, b_{2}, \ldots$

$$
r_{3}=0.82915 r_{1}^{3}-0.000434734 r_{1} \dot{r}_{1}^{2}
$$

In order to convert the modal coordinates back to physical coordinates we use Eq. (9). Therefore, in physical coordinates,

$$
\left[\begin{array}{l}
u_{1} \\
u_{2} \\
u_{3} \\
u_{4}
\end{array}\right]=\left[\begin{array}{c}
-r_{1}-0.82915 r_{1}^{3}+0.000434734 r_{1} \dot{r}_{1}^{2} \\
-0.318385 r_{1}+0.0453118 r_{1}^{3}-0.0000237575 r_{1} \dot{r}_{1}^{2} \\
0 \\
r_{1}+0.82915 r_{1}^{3}-0.000434734 r_{1} \dot{r}_{1}^{2}
\end{array}\right]
$$

Knowing these four nodal displacements we approximate the continuous mode shape using the shape functions of the linear Euler-Bernoulli beam

This process was repeated for models with greater refinement. In each case, all of the linear modes available the corresponding linear finite element model were used in the solution for the nonlinear normal modes. For convergence as a function of modes used (using a continuum Galerkin solution), the reader is referred to the work by Pesheck et al. [18].

\section{Results}

Several solutions of the resulting models were carried out for a wide range of amplitudes. The first and third mode shapes were obtained using the methods described in the previous section using two, four, eight and sixteen elements. The results clearly illustrate the convergence of the method to the continuous solution as presented by Boivin, Pierre, and Shaw [3] as the number of elements is increased. The convergence of the continuous solution to the numerical solution, computed by direct integration of the set of nonlinear, coupled, first-order ODE's has already been demonstrated in [3]. As is the case for linear systems, the periodicity of the time response converged here much more quickly than the (nonlinear) mode shapes. Maximum error in the time history occurred at the peak amplitudes. Those results are illustrated in this work, although these results are obtained from analytical solution for the nonlinear modes. Time histories of numerical simulations show much faster convergence than the mode shapes and are presented in [20].

The comparisons are made by determining the modes of the FEM and continuous models at matching strain energy levels (zero kinetic energy) and observing the difference. The strain energy in the beam is given by [5] as

$$
\Lambda_{\text {beam }}=\begin{gathered}
E I \\
2
\end{gathered} \int_{0}^{L}\left(\begin{array}{c}
d^{2} u \\
d s^{2}
\end{array}\right)^{2} d s
$$


Since we have defined

$$
\alpha=\begin{gathered}
E I \\
m
\end{gathered}
$$

we can calculate the energy of the beam per unit mass as,

$$
\Lambda_{\text {beam }}={ }_{2}^{\alpha} \int_{0}^{L}\left(\begin{array}{c}
d^{2} u \\
d s^{2}
\end{array}\right)^{2} d s
$$

For the beam with the non-linear spring attached to it the energy of the spring must be taken into account. This energy is a function of the spring deflection and is calculated as

$$
\Lambda_{\text {spring }}=\int_{0}^{\substack{\left.u\right|_{2} \\ \text { d }}} F(u) d u
$$

where

$$
F(w)=\left.\gamma u^{3}\right|_{s={ }_{2}^{l}}
$$

is the spring force for a displacement $u\left(\begin{array}{l}l \\ 2\end{array}\right)$, so that the energy is given by

$$
\Lambda_{\text {spring }}=\left.\begin{gathered}
\gamma u^{4} \\
4
\end{gathered}\right|_{l / 2}
$$

Since $\underset{m}{\gamma}=\beta$, the energy of the spring per unit mass is

$$
\Lambda_{\text {spring }}={ }_{4}^{1} u\left(\begin{array}{l}
l \\
2
\end{array}\right)^{4} \beta
$$

Therefore the total energy for the non-linear beam is calculated as,

$$
\Lambda=\Lambda_{\text {beam }}+\Lambda_{\text {spring }}
$$

Once energy amplitudes are matched, the an energy normalized error as a function of $x$ is calculated as

$$
\epsilon(s)=u(s)_{\text {linear }}-u(s)_{\text {nonlinear }}
$$

Thus the results show the mode shapes of each model for the same amount of energy. At low energies the nonlinear beam mode is fairly close to the its linear counterpart. However, as the energy input to the system is increased, the effect of the non-linear spring becomes more apparent. It may be noted that a change in the energy input to the system can also be translated to a change in the initial amplitude of the system as in [3] where a numerical solution in time is implemented. That work further details the shape the modes themselves and thus they are not repeated here. Here, the modes from the different methods have been compared on the basis of their energies because this provides a better feel for the system and is possibly a more practical way of looking at the results because "amplitude" is a subjective term and depends on a predefined notion of the mode shape.

The results show that a fair approximation of the beam dynamics of the first mode at low amplitudes are obtained by using two elements. On increasing the amplitude the nonlinearity becomes more evident and the 2 element solution deviates from the continuous solution. The beam dynamics can be obtained with better accuracy by increasing the number of elements. However, at least four elements are required to somewhat capture the third mode and eight elements are necessary to show acceptable agreement.

The error between the continuous and finite element methods is shown versus position along the length of the beam in Figs 3(a)-4(d). The error increases rapidly as the amplitude approaches 1. On the other hand, the normalized error can be expected to remain constant for the linear system. A quick comparison of the error plots also shows how, as expected, the magnitude of the normalized error decreases with an increase in the number of elements. This is true for the first as well as the third mode shapes.

The contribution of higher linear modes to the nonlinear modes can also be observed in the plots. The two element solution appears to be more accurate than the four element solution for the third mode for a wide range of amplitudes. 


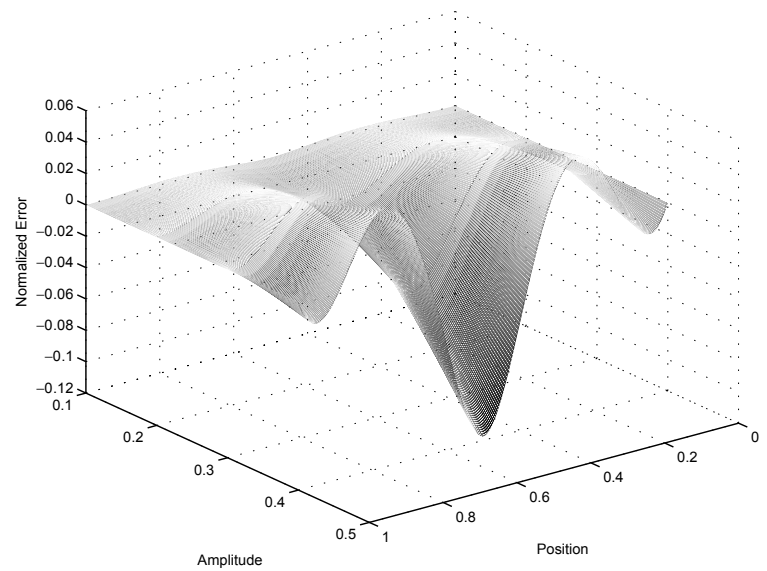

(a) Normalized error plot, 2-elements, mode 1

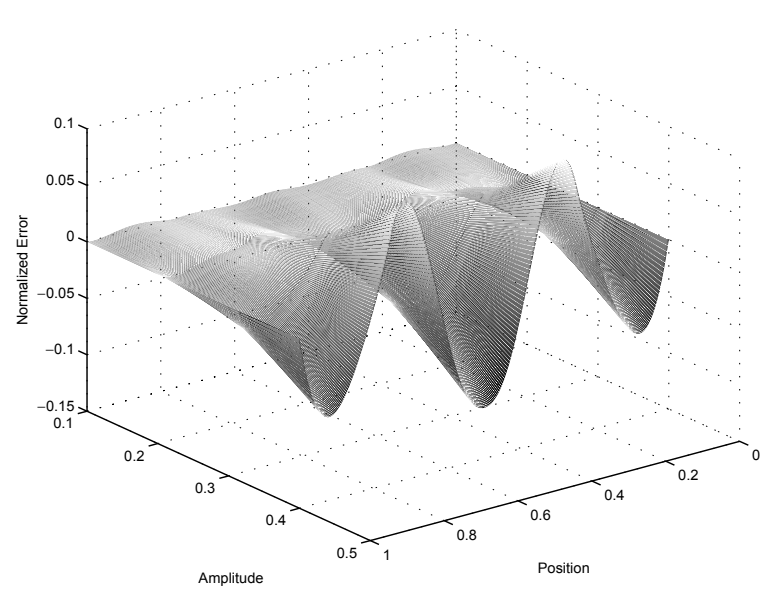

(c) Normalized error plot, 8-elements, mode 1

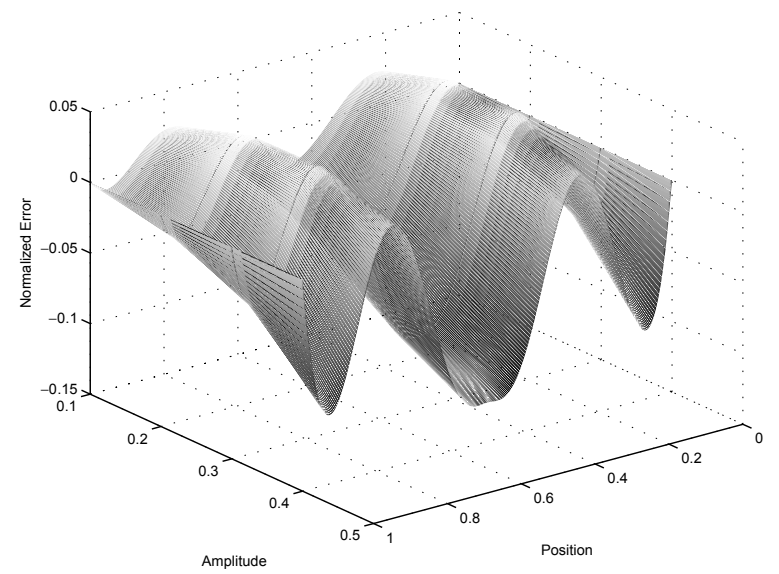

(b) Normalized error plot, 4-elements, mode 1

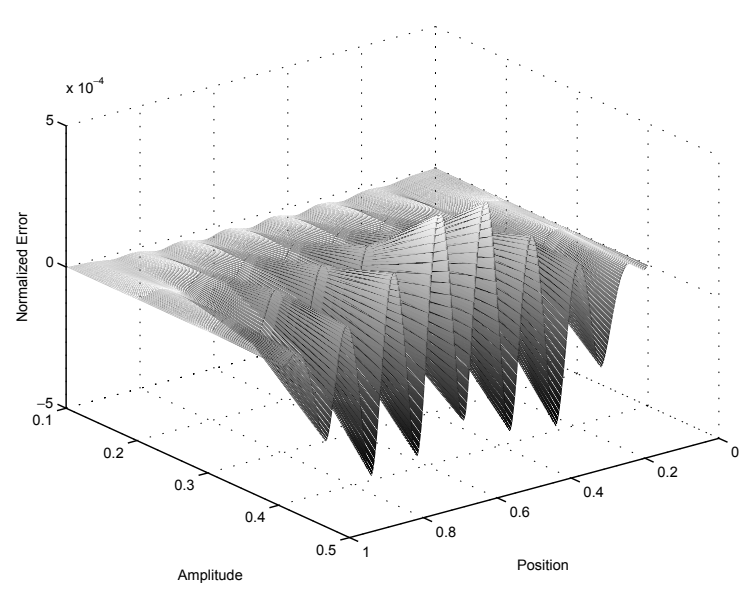

(d) Normalized error plot, 16-elements, mode 1

Fig. 3. Normalized errors in mode 1.

However closer examination of the results proves this to be an aberration. Even at low amplitudes, the two element formulation is not capable of representing the third nonlinear normal mode (Fig. 4(a)). At low amplitudes the four element formulation is clearly more accurate than the two element solution (Fig. 4(b)). The contribution of the fifth linear mode is clearly imperative to representing the third nonlinear normal mode at higher amplitude as is illustrated in Fig. 4(c) where the high amplitude error in the modal representation is less than $0.5 \%$. Even for linear systems one does not use a four degree of freedom system to find the third mode, so the four element model should not be expected to yield good results. In order to obtain reasonable accuracy for $n$ modes, a 2-D finite element frame model generally must have $2 n$ degrees of freedom.

It is clear from the example considered here that more degrees of freedom need to be used for nonlinear systems than for comparable linear systems in order to successfully represent the nonlinear modes. Table 1 shows the results of the convergence study conducted on the example beam considered here. Convergence is defined as a maximum of 5\% normalized error in the mode shape. Recall that for a linear system convergence is not affected by a change in the amplitude. Figures 3(a) through 4(d) explicitly show the errors in the determined mode shapes, normalized with respect to the modal amplitude, as a function of modal amplitude and number of elements. Although the nonlinear modes require more elements to accurately represent them than their linear counterparts, that number does not appear to continue to increase with greater amplitude. 
Table 1

Convergence study: Number of elements needed for reasonable (subjective) accuracy

\begin{tabular}{ccccc} 
& \multicolumn{2}{c}{ Linear } & \multicolumn{2}{c}{ Nonlinear } \\
Amplitude & Mode 1 & Mode 3 & Mode 1 & Mode 3 \\
0.1 & 2 & 4 & 2 & 4 \\
0.2 & 2 & 4 & 4 & 4 \\
0.3 & 2 & 4 & 4 & 8 \\
0.4 & 2 & 4 & 4 & 8
\end{tabular}

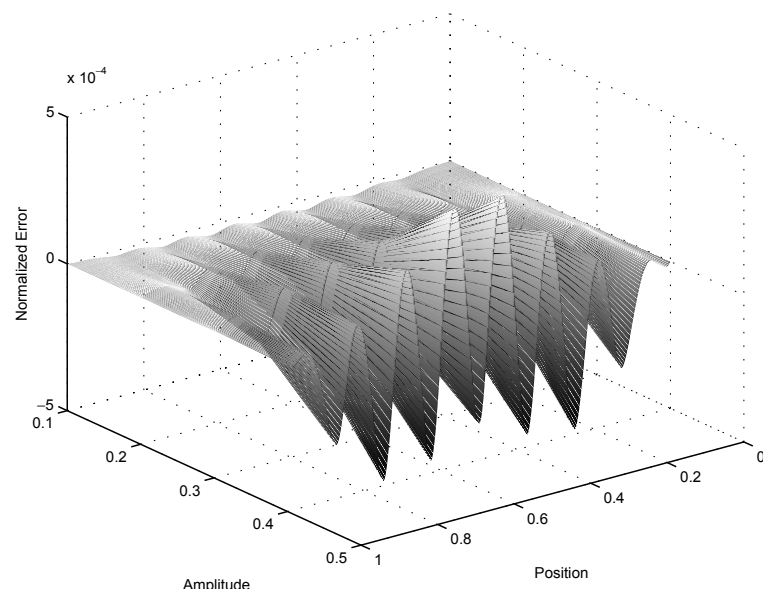

(a) Normalized error plot, 2-elements, mode 3

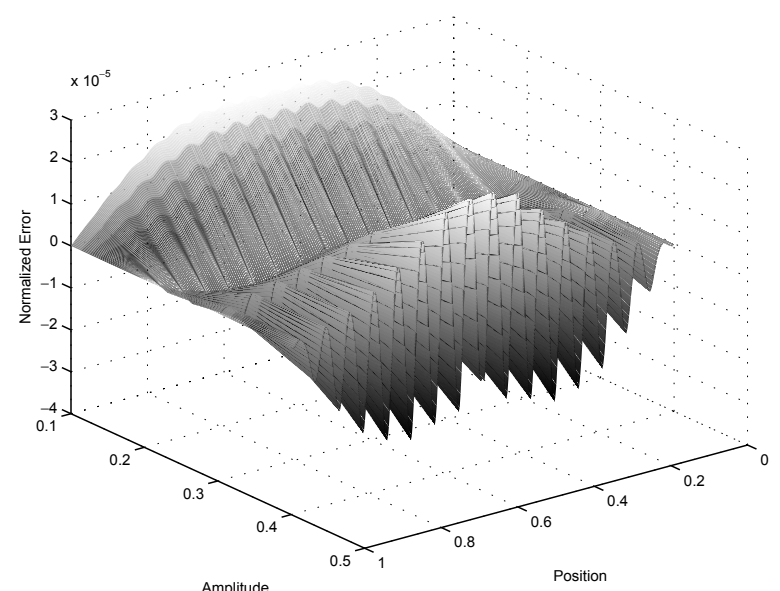

(a) Normalized error plot, 8-elements, mode 3

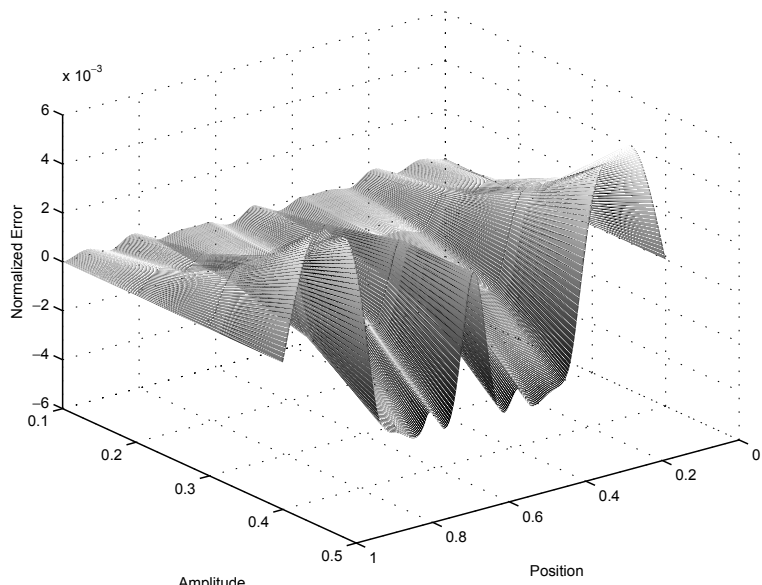

(b) Normalized error plot, 4-elements, mode 3

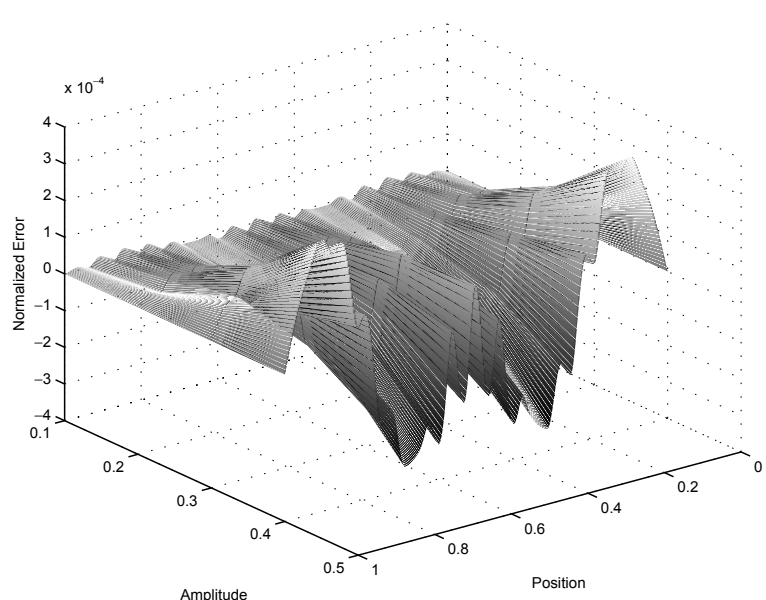

(b) Normalized error plot, 16-elements, mode 3

Fig. 4. Normalized errors in mode 3.

\section{Conclusion}

The convergence of a finite element model of a simply supported beam with a cubic nonlinear spring has been demonstrated. Results show the expected convergence of the finite element model, but also show that the solution can diverge at high amplitudes with the addition of elements when the number of elements is still insufficient for adequate modal representation. Also demonstrated is that while more elements are needed to accurately represent the modes of the nonlinear system, the number of elements needed does not continue to increase monotonically with 
amplitude. While the results presented are specific to this example, they clearly indicate that the convergence of nonlinear finite element models introduces new complexities to the dynamic analyst.

\section{References}

[1] S.W. Shaw and C. Pierre, On Nonlinear Normal Modes, Proceedings of the Winter Annual Meeting of the A.S.M.E. 50 (1992), 1-5.

[2] S.W. Shaw and C. Pierre, Normal Modes for Nonlinear Vibratory Systems, Journal of Sound and Vibration 164(1) (1993), 85-124.

[3] N. Boivin, C. Pierre and S.W. Shaw, Nonlinear Normal Modes, Invariance, and Modal Dynamics Approximations of Nonlinear Systems, Nonlinear Dynamics 8(3) (October 1995), 315-346.

[4] P. Ribeiro, Nonlinear Vibrations of Simply-Supported Plates by the P-Version Finite Element Method, Finite Elements in Analysis and Design 41 (May 2005), 911-924.

[5] L. Meirovitch, Analytical Methods in Vibrations, Macmillan, 1967.

[6] S.P. Timoshenko, W. Weaver and D.H. Young, Vibration Problems in Engineering, Wiley, 1990.

[7] R.M. Rosenberg, The Normal Modes of Nonlinear T-Degree of Freedom Systems, Journal of Applied Mechanics 30 (March 1962), 7-14.

[8] L.I. Manevich and I.V. Mikhlin, On Periodic Solutions Close to Rectilinear Normal Vibration Modes, PMM 36 (1972), 1051-1058.

[9] M.E. King and A.F. Vakakis, An Energy-Based Approach to Computing Resonant Nonlinear Normal Modes, Journal of Applied Mechanics 63 (1996), 810-819.

[10] A.H. Nayfeh and S.A. Nayfeh, On Nonlinear Modes of Continuous Systems, Journal of Vibration and Acoustics 116(1) (1994), $129-136$.

[11] A.F. Vakakis and R.H. Rand, Normal Modes and Global Dynamics of a Two-Degree-Of-Freedom Nonlinear System - I. Low Energies, International Journal of Non-Linear Mechanics 27(5) (1992), 861-874.

[12] S.W. Shaw and C. Pierre, Normal Modes of Vibration for Nonlinear Continuous Systems, Journal of Sound and Vibration 169(3) (1994), 319-347.

[13] S.W. Shaw, An Invariant Manifold Approach to Nonlinear Normal Modes of Oscillation, Journal of Sound and Vibration 4(5) (SeptemberOctober 1994), 419-448.

[14] J. Carr, Applications of Centre Manifold Theory, Springer Verlag, 1981.

[15] A.F. Vakakis, Normal modes and localization in nonlinear systems, A.F. Vakakis et al., John Wiley, New York: c1996, Includes bibliographical references (pp. 517-547) and index.

[16] A.H. Nayfeh, On direct methods for constructing nonlinear normal modes of continuous systems, Journal of Vibration and Control 1 (1995), 389-430.

[17] P. Apiwattanalunggarn, S.W. Shaw, C. Pierre and D.Y. Jiang, Finite-element-based nonlinear modal reduction of a rotating beam with large-amplitude motion, Journal of Vibration and Control 9 (March 2003), 235-263.

[18] E. Pesheck, C. Pierre and S.W. Shaw, A new Galerkin-based approach for accurate non-linear normal modes through invariant manifolds, Journal of Sound and Vibration 249 (31 Jan 2002), 971-993.

[19] R.D. Cook, D.S. Malkus and M.E. Plesha, Concepts and Applications of Finite Element Analysis, 3rd ed., John Wiley \& Sons, New York, 1989.

[20] R. Balagangadhar, On the Convergence of Nonlinear Normal Modes Using the Finite Element Method, Master's thesis, Wright State University, 1997. 

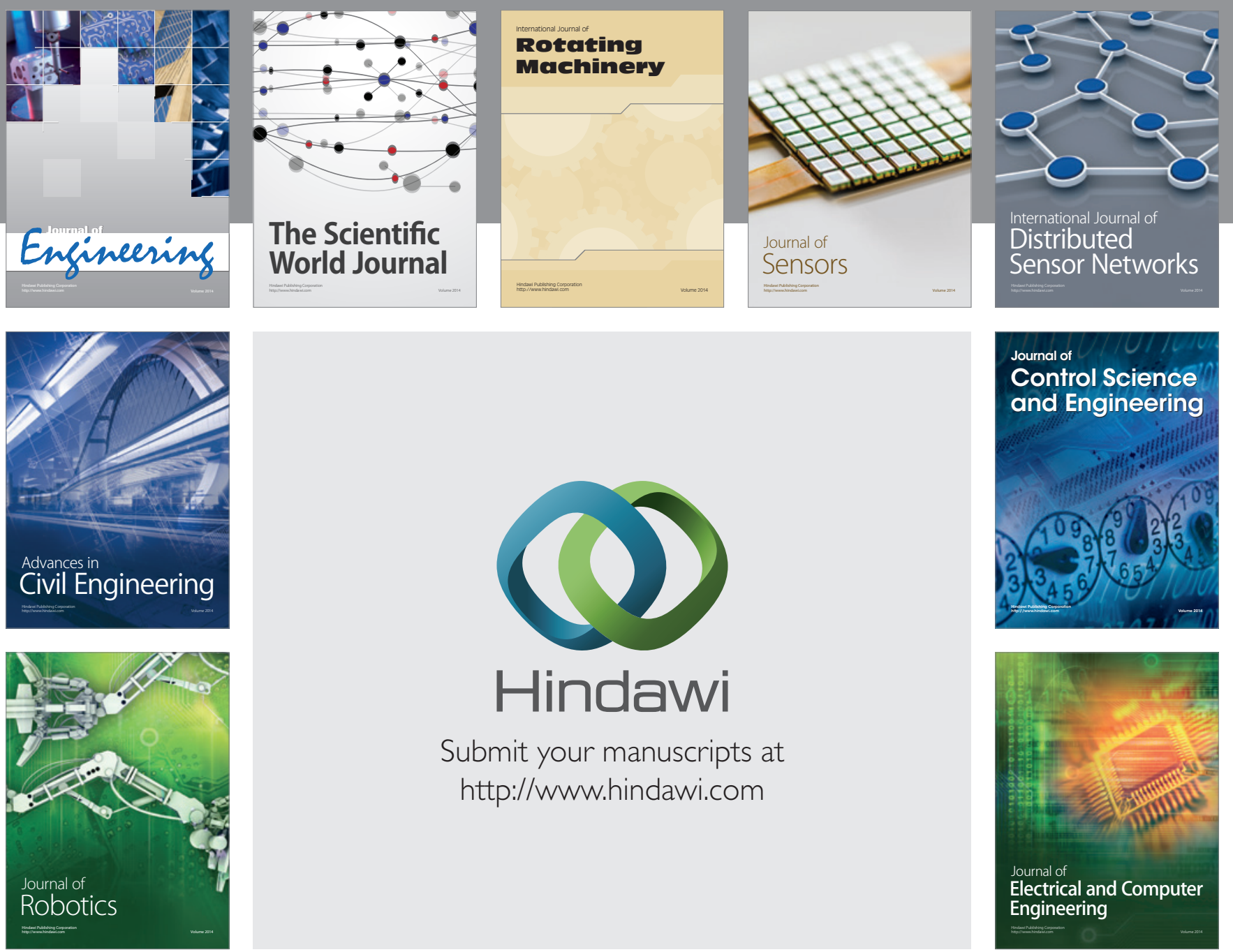

Submit your manuscripts at

http://www.hindawi.com
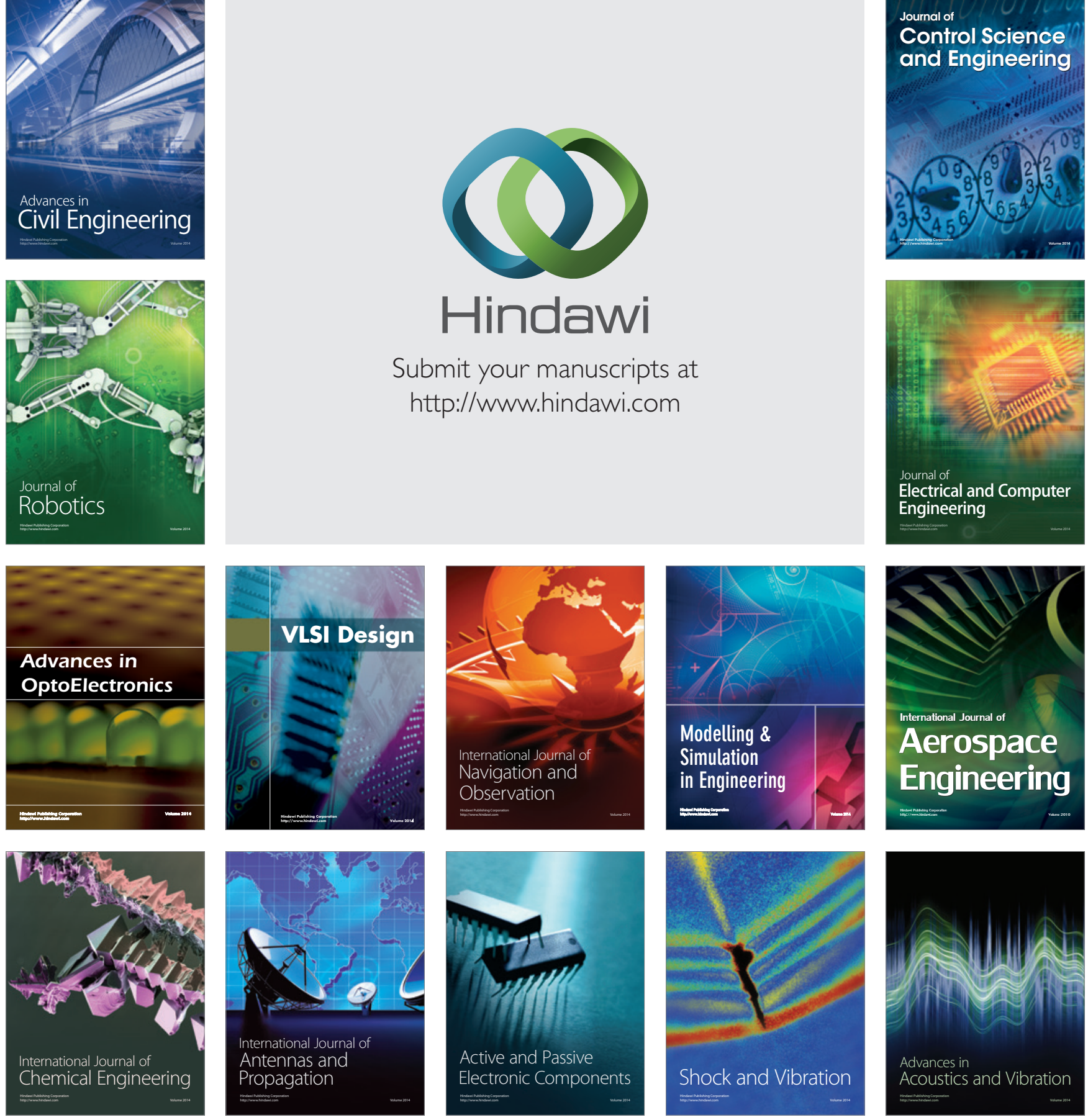